\title{
Theory of Radiation From Sources Immersed in Anisotropic Media
}

\author{
James R. Wait \\ Contribution From the Central Radio Propagation Laboratory, National Bureau of Standards, \\ Boulder, Colo.
}

(May 20, 1964)

\begin{abstract}
The electromagnetic fields produced by an electric dipole immersed in an anisotropic medium are considered. Various approaches to the problem are outlined with special reference to a cold plasma. An attempt is made to show the close relationship between previously published work on this subject. It is shown that information on the radiation field in an anisotropic media may be obtained directly from the shape of the refractive index surface.
\end{abstract}

\section{Introduction}

There is now a vast amount of literature on the subject of electromagnetic waves in anisotropic media. However, until quite recently, only plane wave solutions have been considered in any detail. In most applications to the ionosphere, this approach has been justified since the transmitting and receiving antennas were ground based. In recent years, the situation is gradually changing since, increasingly often, the source is placed within the anisotropic medium. The best example is when a transmitter is located in a satellite which has an antenna attached to it in some manner.

It is the purpose of this paper to review some of the recent theoretical investigations on the subject of radiation from sources in anisotropic media. It is assumed throughout that nonlinear phenomena may be ignored. Thus, the medium is characterized by a tensor dielectric constant and a tensor magnetic permeability whose elements are independent of the field.

In the first part of this paper, the Green's function for a generally anisotropic (i.e., gyrotropic) media is considered. The results are then specialized to a medium whose magnetic properties are isotropic. This important special case corresponds to a cold plasma which may be described as a gyroelectric medium. Using this type of medium as an example, some alternative approaches to solving for the fields are described. The extensions required to treat stratified anisotropic media are also described briefly. Finally, the special problems associated with asymptotic evaluation of the integrals are considered.

\section{Green's Functions for Generally Anisotropic Medium}

The present development of the Green's function is closely related to that of Bunkin [1957], Kogelnik [1960], Meecham [1961], Kuehl [1962], and Ament [1963]. In particular, following Ament [1963], we assume a Cartesian coordinate system $\left(x_{1}, x_{2}, x_{3}\right)$ in which $E_{j}, H_{j}$, and $J_{j}$ represent the component of the electric field, magnetic field and source current density, parallel to the $x_{j}$ axis. As usual, all field quantities vary according to $\exp (i \omega t)$.

The medium itself is considered to be homogeneous and generally anisotropic. Thus, the dielectric constant and magnetic permeability are denoted by tensors $(\epsilon)$ and $(\mu)$, independent of position. For this situation, Maxwell's equations for the $j$ th component are written

$$
(\vec{\nabla} \times \vec{E})_{j}+i \omega \mu_{j n} H_{n}=0
$$




$$
(\vec{\nabla} \times \vec{H})_{j}-i \omega \epsilon_{j n} E_{n}=J_{j}
$$

where $\mu_{j n}=(\mu)$ and $\epsilon_{j n}=(\epsilon)$ are three-by-three matrices or dyads with constant elements.

Formally, the field $E_{i}(\overrightarrow{x)}$ at the vector position $\vec{x}$ may be obtained via a Green's function $\tilde{G}_{i j}\left(\vec{x}, \overrightarrow{x^{\prime}}\right)$ from the current density $J_{j}\left(\overrightarrow{x^{\prime}}\right)$ at vector position $\overrightarrow{x^{\prime}}$. One should note that the components of $\vec{x}$ are $x_{1}, x_{2}, x_{3}$ and those of $\overrightarrow{x^{\prime}}$ are $x_{1}^{\prime}, x_{2}^{\prime}, x_{3}^{\prime}$. The Green's function $\tilde{G}_{i j}$ is a tensor and is a function of both $\vec{x}$ and $\overrightarrow{x^{\prime}}$. It is defined by the relation

$$
E_{i} \overrightarrow{(x)}=\iiint \tilde{G}_{i j}\left(\vec{x}, \overrightarrow{x^{\prime}}\right) J_{j}\left(\overrightarrow{x^{\prime}}\right) d x_{1}^{\prime} d x_{2}^{\prime} d x_{3}^{\prime}
$$

where the integration is over any volume which encloses the source current density. A more compact description of (3) is written

$$
\left.E_{i}(\vec{x})=\int G_{i j} \overrightarrow{(x,} \cdot \overrightarrow{x^{\prime}}\right) J_{j}\left(\overrightarrow{x^{\prime}}\right) d^{3} x^{\prime}
$$

To obtain an expression for the Green's function, it is convenient to write Maxwell's equations in symbolic form. For example, if $\partial_{i}$ symbolizes the derivative $\partial / \partial x_{i}$, it is evident that

$$
\vec{\nabla} \times \vec{E}=-i(\mu) \omega \vec{H}
$$

is equivalent to

$$
\left(\begin{array}{rrr}
0 & -\partial_{3} & \partial_{2} \\
\partial_{3} & 0 & -\partial_{1} \\
-\partial_{2} & \partial_{1} & 0
\end{array}\right)\left[\begin{array}{l}
E_{1} \\
E_{2} \\
E_{3}
\end{array}\right]=-i \omega\left(\begin{array}{lll}
\mu_{11} & \mu_{12} & \mu_{13} \\
\mu_{21} & \mu_{22} & \mu_{23} \\
\mu_{31} & \mu_{32} & \mu_{33}
\end{array}\right)\left[\begin{array}{c}
H_{1} \\
H_{2} \\
H_{3}
\end{array}\right]
$$

or, simply

$$
\tilde{\partial} \cdot \vec{E}=-i \omega \tilde{\mu} \cdot \vec{H}
$$

Similarly, the other of Maxwell's equations,

$$
\vec{\nabla} \times \vec{H}=i(\epsilon) \omega \vec{E}+\vec{J}
$$

becomes

$$
\tilde{\partial} \cdot \vec{H}=i \omega \tilde{\epsilon} \cdot \vec{E}+\vec{J}
$$

The vector $\vec{H}$ may now be eliminated from (6) and (8) to obtain

$$
\tilde{\partial} \cdot \tilde{\nu} \cdot \tilde{\partial} \cdot \vec{E}-\omega^{2} \tilde{\epsilon} \cdot \vec{E}=-i \omega \vec{J}
$$

where

$$
\tilde{\nu}=\frac{1}{\tilde{\mu}} \text { is the matrix inverse to } \tilde{\mu} \text {. }
$$


To facilitate the solution of (9), it is assumed that both $\vec{E}$ and $\vec{J}$ have three-dimensional Fourier representations. These may be regarded as superpositions of plane waves propagating as exp $\left[-i k_{1} x_{1}-i k_{2} x_{2}-i k_{3} x_{3}\right]=\exp [-\overrightarrow{i k} \cdot \vec{x}]$ where $\vec{k}$ is the (vector) wave number. Thus,

$$
e_{n}(\vec{k})=(2 \pi)^{-3} \int E_{n}(\vec{x}) \cdot e^{+\overrightarrow{i k} \cdot \vec{x}} d^{3} x
$$

is the transform of $E_{n}(\overrightarrow{x)}$ when $n=1,2$, or 3 . The inverse transform is given by

$$
E_{n}(\vec{x})=\int e_{n}(\vec{k}) e^{-\overrightarrow{i k} \cdot \vec{x}} d^{3} k
$$

which is a volume integral over. $k$ space. The transform of $J_{n}(\vec{x})$, which is denoted by $j_{n}(\vec{k})$, is defined in the same fashion.

The differentiations in (9), indicated by the $\tilde{\partial}$ tensors, may be carried out under the integral signs. Then, on equating coefficients of $\exp (-\overrightarrow{i k} \cdot \vec{x})$, we obtain

$$
\tilde{K} \cdot \tilde{\nu} \cdot \tilde{K} \cdot \vec{e}+\omega^{2} \tilde{\epsilon} \cdot \vec{e}=i \omega \vec{j}
$$

where $\tilde{K}$ represents the matrix

$$
\left(\begin{array}{rrr}
0 & -k_{3} & k_{2} \\
k_{3} & 0 & -k_{1} \\
-k_{2} & k_{1} & 0
\end{array}\right) .
$$

Equation (12) may be solved formally to yield

$$
\vec{e}(\vec{k})=i \omega \tilde{M} \cdot \vec{j}(\vec{k})
$$

where

$$
\tilde{M}=\frac{1}{\tilde{K} \cdot \tilde{\nu} \cdot \tilde{K}+\omega^{2} \tilde{\epsilon}}
$$

From (11) we then find that

$$
\begin{aligned}
\vec{E}(\vec{x}) & =i \omega \int e^{-\overrightarrow{i k} \cdot \vec{x}} \tilde{M} \cdot \vec{j}\left(\overrightarrow{k)} d^{3} k\right. \\
& =i \omega \int e^{-\overrightarrow{i k} \cdot \vec{x}} \tilde{M} \cdot \int J\left(\overrightarrow{x^{\prime}}\right) e^{+\overrightarrow{i k} \cdot \overrightarrow{x^{\prime}}}(2 \pi)^{-3} d^{3} x^{\prime} d^{3} k
\end{aligned}
$$

This result can be written in the required form

$$
\vec{E}(\vec{x})=\int \tilde{G}\left(\vec{x}, \overrightarrow{x^{\prime}}\right) \cdot \vec{J}\left(\overrightarrow{x^{\prime}}\right) d^{3} x^{\prime}
$$

if

$$
\tilde{G}\left(\vec{x}, \overrightarrow{x^{\prime}}\right)=\frac{i \omega}{(2 \pi)^{3}} \int e^{-\overrightarrow{i k} \cdot\left(\vec{x}-\overrightarrow{x^{\prime}}\right)} \tilde{M} d^{3} k
$$

and provided the order of the $x^{\prime}$ and the $k$ integration may be interchanged. In matrix notation, 
the Green's function is written

$$
G_{i j}\left(\vec{x}, \overrightarrow{x^{\prime}}\right)=\frac{i \omega}{(2 \pi)^{3}} \int e^{-\overrightarrow{i k} \cdot\left(\vec{x}-\overrightarrow{x^{\prime}}\right)} M_{i j} d^{3} k
$$

which involves the $\vec{k}$ - dependent matrix $M_{i j}$.

It may be easily shown that $M_{i j}$ is an even function of $\vec{k}$ in that it has the same value when $\vec{k}$ (or $k_{1}, k_{2}, k_{3}$ ) is replaced by $-\vec{k}$ (or by $-k_{1},-k_{2},-k_{3}$ ). Thus, on noting that $M_{i j}$ is an explicit function of $\left(\vec{x}-\vec{x}^{\prime}\right)$ and $\vec{k}$, it is seen that

$$
G_{i j}\left(\vec{x}-\overrightarrow{x^{\prime}}\right)=G_{i j} \overrightarrow{x^{\prime}}-\overrightarrow{x)}
$$

\section{Green's Function for Gyroelectric Medium}

The development of the Green's function as given above is applicable to homogeneous but generally anisotropic media. The important special case is when the magnetic properties are isotropic. In this instance,

$$
(\tilde{\mu})=\left(\begin{array}{ccc}
\mu_{0} & 0 & 0 \\
0 & \mu_{0} & 0 \\
0 & 0 & \mu_{0}
\end{array}\right),
$$

where $\mu_{0}$ is the usual magnetic permeability of the media. In most ionospheric-type plasma, it is permissible to regard $\mu_{0}$ as the permeability of free space. However, this is not an essential restriction on the theory.

A further simplification may be made if the d-c magnetic field is directed along the $z$ axis of the Cartesian coordinate system. The tensor dielectric constant then has the form

$$
(\tilde{\epsilon})=\left(\begin{array}{rrr}
\epsilon & -i g & 0 \\
i g & \epsilon & 0 \\
0 & 0 & \hat{\epsilon}
\end{array}\right),
$$

where the (complex) constants $\epsilon, \hat{\epsilon}$, and $g$ depend on the properties of the medium.

While the specific form of the Green's function for the gyroelectric case may be obtained as a special case, it is instructive to derive it directly following the method of Kogelnik [1960].

The relevant wave equation, corresponding to (9), is now written in vector-dyadic notation,

$$
\left(\vec{\nabla} \vec{\nabla}-\Delta \tilde{1}-\frac{\omega^{2}}{c^{2}} \frac{\tilde{\epsilon}}{\epsilon_{0}}\right) \vec{E}=-i \mu_{0} \omega \vec{J}
$$

where $\Delta=\vec{\nabla} \cdot \vec{\nabla}$ is the Laplacian operator, $\tilde{1}$ is the unit matrix or dyad, and $\vec{\nabla} \vec{\nabla}$ and $\tilde{\epsilon}$ are also dyads. The elements of $\vec{\nabla} \vec{\nabla}$ are the differential operators $\partial^{2} / \partial x_{i} \partial x_{k}$.

Plane wave solutions of (23) have the form

$$
\vec{E}(r)=\overrightarrow{E_{0}} e^{-i \vec{k} \cdot \vec{x}}
$$

Then, since

$$
\frac{\partial}{\partial x_{i}} e^{-i \vec{k} \cdot \vec{x}}=-i k_{i} e^{-i \vec{k} \cdot \vec{x}}
$$

it follows that

$$
\left(\vec{\nabla} \vec{\nabla}-\Delta \tilde{1}-\frac{\omega^{2}}{c^{2}} \frac{\tilde{\epsilon}}{\epsilon_{0}}\right) e^{-i \vec{k} \cdot \vec{x}}=-\tilde{\Omega}(\vec{k}) e^{-i \vec{k} \cdot \vec{x}},
$$


where

$$
\tilde{\Omega}(\vec{k})=\vec{k} \vec{k}-k^{2} \tilde{1}+\frac{\omega^{2}}{c^{2}} \frac{\tilde{\epsilon}}{\epsilon_{0}}
$$

is the "wave matrix." The homogeneous wave equation thus has the form

$$
\tilde{\Omega}(\vec{k}) \vec{E}_{0} e^{-i \vec{k}} \cdot \vec{x}=0 .
$$

The condition for nonvanishing fields is

$$
\operatorname{det} \tilde{\Omega}(\vec{k})=0 \text {, }
$$

where det denotes the determinant of the elements of the tensor. The planes of equal phase are specified by the wave normal $\vec{n}=\left(n_{1}, n_{2}, n_{3}\right)$ such that $\vec{k}=k \vec{n}$. The components of the direction cosines may be expressed in terms of angles $\alpha$ and $\beta$ by

$$
\begin{aligned}
& n_{1}=\sin \alpha \cos \beta, \\
& n_{2}=\sin \alpha \sin \beta, \\
& n_{3}=\cos \alpha .
\end{aligned}
$$

Using the specific form of the dielectric tensor defined above, it is not difficult to show that

$$
\operatorname{det} \tilde{\Omega}(\vec{k})=\mu_{0} \omega^{2}\left(\epsilon \sin ^{2} \alpha+\hat{\epsilon} \cos ^{2} \alpha\right)\left(k^{2}-k_{\mathrm{I}}^{2}\right)\left(k^{2}-k_{\mathrm{II}}^{2}\right)=0,
$$

where

$$
\left.\begin{array}{l}
k_{\mathrm{I}}^{2} \\
k_{\mathrm{II}}^{2}
\end{array}\right\}=\frac{\left(\epsilon^{2}-g^{2}\right) \sin ^{2} \alpha+\epsilon \hat{\epsilon}\left(1+\cos ^{2} \alpha\right) \pm \Gamma}{2\left(c^{2} / \omega^{2}\right)\left(\epsilon \sin ^{2} \alpha+\hat{\epsilon} \cos ^{2} \alpha\right) \epsilon_{0}},
$$

where

$$
\Gamma=\left[\left(\epsilon^{2}-g^{2}-\epsilon \hat{\epsilon}\right)^{2} \sin ^{4} \alpha+4 g^{2} \hat{\epsilon}^{2} \cos ^{2} \alpha\right]^{1 / 2} .
$$

The roots, $k_{\mathrm{I}}$ and $k_{\mathrm{II}}$, of (30), are the (complex) wave numbers for the "ordinary" and the "extraordinary waves. They depend on the angle $\alpha$ between the wave normal and the d-c magnetic field.

Rather than using a Fourier representation to obtain the Green's function, it is instructive to utilize the properties of Dirac's $\delta$ functions. These are defined, in the usual manner, by

$$
\int_{-\infty}^{+\infty} f\left(x^{\prime}\right) \delta\left(x-x^{\prime}\right) d x^{\prime}=f(x)
$$

and

$$
\delta(x)=\frac{1}{2 \pi} \int_{-\infty}^{+\infty} e^{-i k_{1} x} d k_{1} .
$$

Then, if

$$
\vec{E}(r)=\int \tilde{G}\left(\vec{x}, \overrightarrow{x^{\prime}}\right) \cdot \vec{J}\left(x^{\prime}\right) d^{3} x^{\prime},
$$


it follows that $\tilde{G}$ must satisfy

$$
\left(\vec{\nabla} \vec{\nabla}-\Delta \tilde{1}-\frac{\omega^{2}}{c^{2}} \frac{\tilde{\epsilon}}{\epsilon_{0}}\right) \tilde{G}\left(\vec{x}, \overrightarrow{x^{\prime}}\right)=-i \mu_{0} \omega \tilde{1} \delta\left(\vec{x}-\overrightarrow{x^{\prime}}\right),
$$

where

$$
\delta(\vec{x})=\delta\left(x_{1}\right) \delta\left(x_{2}\right) \delta\left(x_{3}\right)
$$

This step may be readily verified if both sides of the equation are multiplied by $\vec{J}$ and then performing the volume integration. It should be noted that $\vec{\nabla} \vec{\nabla}$ and $\Delta$ operate on the variables $\vec{x}$ and not $\vec{x}$.

To complete the derivation, (26) is rewritten in the form

$$
\left(\vec{\nabla} \vec{\nabla}-\Delta \tilde{\mathrm{l}}-\frac{\omega^{2}}{c^{2}}\right) \tilde{\Omega}^{-1} e^{-i \vec{k}} \cdot \vec{x}=-\tilde{\mathrm{l}} e^{-i \vec{k} \cdot \vec{x}},
$$

where $\tilde{\Omega}^{-1}$ is the inverse of the "wave matrix." Both sides of this equation are now multiplied by $\exp \left(\overrightarrow{i k} \cdot \overrightarrow{x^{\prime}}\right)$ and the integrations $\int d^{3} k=\iiint d k_{1} d k_{2} d k_{3}$ are performed. On identifying this equation with (36), it readily follows that

$$
\tilde{G}\left(\vec{x}, \overrightarrow{x^{\prime}}\right)=\frac{i \mu_{0} \omega}{(2 \pi)^{3}} \int \tilde{\Omega}^{-1} e^{-i \vec{k} \cdot\left(\vec{x}-\vec{x}^{\prime}\right)} d^{3} k,
$$

which establishes the desired result. As indicated, this is a special case of (19) for the generally anisotropic medium. Equation (39) was also given by Bunkin [1957] in slightly modified form.

The explicit form for the inverse of $\tilde{\Omega}$ is obtained from Cramer's rule; thus

$$
\tilde{\Omega}^{-1}=\frac{\tilde{\Lambda}}{\operatorname{det} \tilde{\Omega}}
$$

where the matrix $\tilde{\Lambda}$ is the "adjoint" of $\tilde{\Omega}$. For a gyroelectric medium of the kind described, it is found that

$$
\tilde{\Lambda}(\vec{k})=k^{2} \vec{k} \vec{k}=k^{2} \frac{\omega^{2}}{c^{2}} \tilde{\mathscr{L}}+\frac{\omega^{4}}{c^{4}} \tilde{\tilde{\zeta}}
$$

where $\tilde{\mathscr{E}}$ is the adjoint of $\tilde{\boldsymbol{\epsilon}}$, given explicitly by

$$
\tilde{\mathscr{C}} \epsilon_{0}^{2}=\left(\begin{array}{ccl}
\epsilon \hat{\epsilon} & i g \hat{\epsilon} & 0 \\
-i g \hat{\epsilon} & \epsilon \hat{\epsilon} & 0 \\
0 & 0 & \epsilon^{2}-g^{2}
\end{array}\right)
$$

and $\tilde{f} \epsilon_{0}=$

$$
\left(\begin{array}{lcl}
\epsilon\left(n_{1}^{2}+n_{2}^{2}\right)+\hat{\epsilon}\left(n_{1}^{2}+n_{3}^{2}\right) & -i g\left(n_{1}^{2}+n_{2}^{2}\right)+\hat{\epsilon} n_{1} n_{2} & \epsilon n_{1} n_{3}-i g n_{2} n_{3} \\
i g\left(n_{1}^{2}+n_{2}^{2}\right)+\hat{\epsilon} n_{1} n_{2} & \epsilon\left(n_{1}^{2}+n_{2}^{2}\right)+\hat{\epsilon}\left(n_{2}^{2}+n_{3}^{2}\right) & \epsilon n_{2} n_{3}+i g n_{1} n_{3} \\
\epsilon n_{1} n_{3}+i g n_{2} n_{3} & \epsilon n_{2} n_{3}-i g n_{1} n_{3} & \epsilon\left(1+n_{3}^{2}\right)
\end{array}\right)
$$




\section{Modified Field Solution}

A three-dimensional transform technique for solving the dipole problem has also been given by Mittra and Deschamps [1963]. Their results have a number of unique features which should be mentioned. In particular, some ingenious manipulations are made in order to carry out the inversion of the transforms. Furthermore, their final expressions are not restricted to the far zone or to a lossless medium.

The starting point, in Mittra's and Deschamps' analysis, is Maxwell's equations written for impressed electric current sources $\vec{J}$ and the tensor dielectric constant $\tilde{\epsilon}$ as given in (22) while the permeability $\mu_{0}$ is assumed to be that of free space. The eliminating $\vec{H}$ from Maxwell's equations leads readily to the usual inhomogeneous wave equation for $\vec{E}$ given by

$$
\vec{\nabla} \times \vec{\nabla} \times \vec{E}-\mu_{0} \omega^{2} \tilde{\epsilon} \vec{E}=-i \mu_{0} \omega \vec{J}
$$

A coordinate system is now introduced which diagonalizes the tensor $\tilde{\epsilon}$. The unit vectors in this system are denoted $\hat{U}_{1}, \hat{U}_{2}$, and $\hat{U}_{3}$, and are defined by

$$
\hat{U}_{1}=2^{-1 / 2}(\hat{x}+i \hat{y}), \hat{U}_{2}=2^{-1 / 2}(\hat{x}-i \hat{y}), \text { and } \hat{U}_{3}=\hat{z},
$$

in terms of the unit vectors $\hat{x}, \hat{y}$, and $\hat{z}$ in the conventional Cartesian system. It is understood that the d-c magnetic field remains oriented in the axial $\hat{z}$ or $\hat{U}_{3}$ direction. An arbitrary vector $\vec{F}$ may be written in the $U$-system as

$$
\vec{F}=\hat{U}_{1} F_{1}+\hat{U}_{2} F_{2}+\hat{U}_{3} F_{3},
$$

where

$$
F_{1}=\vec{F} \cdot \hat{U}_{2}, F_{2}=\vec{F} \cdot \hat{U}_{1} \text {, and } F_{3}=\vec{F} \cdot \vec{U}_{3}
$$

In terms of the components $F_{x}, F_{y}$, and $F_{z}$ in the conventional Cartesian system, it is seen that

$$
F_{1}=2^{-1 / 2}\left(F_{x}-i F_{y}\right), F_{2}=2^{-1 / 2}\left(F_{x}+i F_{y}\right), \text { and } F_{3}=F_{z} .
$$

The diagonal nature of the $U$-system is immediately evident from the identity

$$
\tilde{\epsilon}=\hat{U}_{1} \hat{U}_{1} \epsilon_{1}+\hat{U}_{2} \hat{U}_{2} \epsilon_{2}+\hat{U}_{3} \hat{U}_{3} \epsilon_{3}
$$

where

$$
\epsilon_{1}+\epsilon+g, \epsilon_{2}=\epsilon-g \text {, and } \epsilon_{3}=\hat{\epsilon} \text {. }
$$

The corresponding expression for the gradient operator in the $U$-system is

$$
\nabla=\hat{U}_{1} d_{1}+\hat{U}_{2} d_{2}+\hat{U}_{3} d_{3}
$$

where the derivative operators are defined by

$$
d_{1}=2^{-1 / 2}\left(\frac{\partial}{\partial x}-i \frac{\partial}{\partial y}\right), d_{2}=2^{-1 / 2}\left(\frac{\partial}{\partial x}+i \frac{\partial}{\partial y}\right), \text { and } d_{3}=\frac{\partial}{\partial z}
$$

After some algebraic manipulations, the inhomogeneous wave equation for $\vec{E}$ may be written in 
convenient matrix form. Thus, in terms of the diagonalizing coordinate system,

$$
\left\{\left(\begin{array}{ccc}
-\left(d_{1} d_{2}+d_{3}^{2}\right) & d_{1}^{2} & d_{1} d_{3} \\
d_{2}^{2} & -\left(d_{1} d_{2}+d_{3}^{2}\right) & d_{2} d_{3} \\
d_{3} d_{2} & d_{1} d_{3} & -2 d_{1} d_{2}
\end{array}\right)-\mu_{0} \omega^{2}\left(\begin{array}{ccc}
\epsilon_{1} & 0 & 0 \\
0 & \epsilon_{2} & 0 \\
0 & 0 & \epsilon_{3}
\end{array}\right)\right\}\left[\begin{array}{c}
E_{1} \\
E_{2} \\
E_{3}
\end{array}\right]=-i \mu_{0} \omega\left[\begin{array}{c}
J_{1} \\
J_{2} \\
J_{3}
\end{array}\right] .
$$

These are matrix equations for the fields, first given in this form by Mittra and Deschamps [1963]. To solve these, it is necessary to employ Fourier representations which transform the differential operators $d_{1}, d_{2}$, and $d_{3}$ to algebraic quantities.

The three-dimensional Fourier transform of $E_{1}(i=1,2,3)$, denoted $\mathscr{E}_{i}(i=1,2,3)$, is defined by

$$
\mathscr{E}_{i}=(2 \pi)^{-3} \int_{-\infty}^{+\infty} \int_{-\infty}^{+\infty} \int_{-\infty}^{+\infty} E_{i}(x, y, z) \exp \left[+i\left(k_{x} x+k_{y} y+k_{z} z\right] d x d y d z .\right.
$$

The differential operators now transform in the following manner

$$
d_{1} \rightarrow 2^{-1 / 2} i\left(k_{x}-i k_{y}\right), d_{2} \rightarrow 2^{-1 / 2} i\left(k_{x}+i k_{y}\right), d_{3} \rightarrow i k_{z} .
$$

For convenience of field calculations, it is desirable to introduce the usual polar form defined by $k_{x}=k \sin \alpha \cos \beta, k_{y}=k \sin \alpha \sin \beta$, and $k_{z}=k \cos \alpha$, whereupon

$$
d_{1} \rightarrow i 2^{-1 / 2} k(\sin \alpha) e^{-i \beta}, d_{2} \rightarrow i 2^{-1 / 2} k(\sin \alpha) e^{i \beta}, d_{3} \rightarrow i k \cos \alpha .
$$

The transform of the matrix equation (52) now has the form

$$
\left\{-\mu_{0} \omega^{2}\left(\begin{array}{ccc}
\epsilon_{1} & 0 & 0 \\
0 & \epsilon_{2} & 0 \\
0 & 0 & \epsilon_{3}
\end{array}\right)+k^{2}\left(\begin{array}{ccc}
-\left(\frac{s^{2}}{2}+c^{2}\right) & \frac{1}{2} s^{2} e^{-2 i \beta} & 2^{-1 / 2} s c e^{-i \beta} \\
\frac{1}{2} s^{2} e^{2 i \beta} & -\left(\frac{s^{2}}{2}+c^{2}\right) & 2^{-1 / 2} s c e^{+i \beta} \\
2^{-1 / 2} s c e^{+i \beta} & 2^{-1 / 2} s c e^{-i \beta} & -s^{2}
\end{array}\right)\right\}\left[\begin{array}{c}
\mathscr{E}_{1} \\
\mathscr{E}_{2} \\
\mathscr{O}_{3}
\end{array}\right]=-i \mu_{0} \omega\left[\begin{array}{c}
\mathscr{I}_{1} \\
\mathscr{I}_{2} \\
\mathscr{I}_{3}
\end{array}\right]
$$

where $s=\sin \alpha$ and $c=\cos \alpha$.

The determinant $\Delta$ of the coefficients inside the curly brackets of (54) is seen to be of the form

$$
\Delta=\mu_{0} \omega^{2}\left(\epsilon \sin ^{2} \alpha+\hat{\epsilon} \cos ^{2} \alpha\right)\left(k^{2}-k_{\mathrm{I}}^{2}\right)\left(k^{2}-k_{\mathrm{II}}^{2}\right),
$$

which is identical to (30) as it must be. The roots of the equation $\Delta=0$ are $k_{\mathrm{I}}$ and $k_{\mathrm{II}}$ and they are given explicitly by (31). As indicated previously, $k_{\mathrm{I}} / k_{0}$ and $k_{\mathrm{II}} / k_{0}$ are the indices of refraction for plane ordinary and extraordinary waves, respectively.

Since the matrix equation (54) involves only algebraic quantities the solution may be obtained directly. Thus, in matrix notation,

$$
[\mathscr{E}]=-i \mu_{0} \omega[A][\mathscr{I}]
$$

where the elements of the inverted matrix are given by

$$
A_{m n}=a_{m n} / \Delta \text {. }
$$

The coefficients $a_{m n}$ are given by

$$
a_{11}=(1 / 2) k^{4} s^{2}-k^{2} \mu_{0} \omega^{2}\left\{\epsilon_{2} s^{2}+\epsilon_{3}\left(\frac{s^{2}}{2}+c^{2}\right)\right\}+\mu_{0} \omega^{4} \epsilon_{3} \epsilon_{2}
$$




$$
\begin{aligned}
& a_{12}=e^{-2 i \beta}(1 / 2) k^{2} s^{2}\left(k^{2}-\mu_{0} \omega^{2} \epsilon_{3}\right), \\
& a_{13}=e^{-i \beta} 2^{-1 / 2} k^{2} s c\left(k^{2}-\mu_{0} \omega^{2} \epsilon_{2}\right), \\
& a_{21}=e^{2 i \beta}(1 / 2) k^{2} s^{2}\left(k^{2}-\mu_{0} \omega^{2} \epsilon_{3}\right), \\
& a_{22}=(1 / 2) k^{4} s^{2}-k^{2} \mu_{0} \omega^{2}\left[\epsilon_{1} s^{2}+\epsilon_{3}\left(\frac{s^{2}}{2}+c^{2}\right)\right]+\mu_{0}^{2} \omega^{4} \epsilon_{1} \epsilon_{3}, \\
& a_{23}=e^{i \beta} 2^{-1 / 2} k^{2} s c\left(k^{2}-\mu_{0} \omega^{2} \epsilon_{1}\right), \\
& a_{31}=e^{i \beta} 2^{-1 / 2} k^{2} s c\left(k^{2}-\mu_{0} \omega^{2} \epsilon_{2}\right), \\
& a_{32}=2^{-1 / 2} e^{-i \beta} k^{2} s c\left(k^{2}-\mu_{0} \omega^{2} \epsilon_{1}\right), \\
& a_{33}=k^{4} c^{2}-\mu_{0} \omega^{2} k^{2}\left(\epsilon_{2}+\epsilon_{1}\right)\left(\frac{s^{2}}{2}+c^{2}\right)+\mu_{0}^{2} \omega^{4} \epsilon_{1} \epsilon_{2} .
\end{aligned}
$$

The explicit evaluation of the three-dimensional inverse transforms remains to be done. The usual approach is to carry out a stationary phase type calculation which is stated to be valid in the far zone. This aspect of the subject is discussed below, but first, it is appropriate to describe the approach by Mittra and Deschamps [1963], which is not restricted to the far field.

To be explicit, an electric dipole source is located at the origin and, to achieve simplicity, the dipole is oriented in the $z$-direction which is parallel to the d-c magnetic field. Thus

$$
\vec{J}=p_{0} \hat{z} \delta(x) \delta(y) \delta(z),
$$

where $\hat{z}$ is a unit vector in the $z$ (or $U_{3}$ ) direction. Thus, from (55), (56), and (57),

$$
\mathscr{E}_{n}=-i \mu_{0} \omega p_{0} \frac{a_{n 3}(k, \alpha, \beta)}{\Delta(k, \alpha)} \text { for } n=1,2,3 \text {, }
$$

and the inverse transform may be written in the form

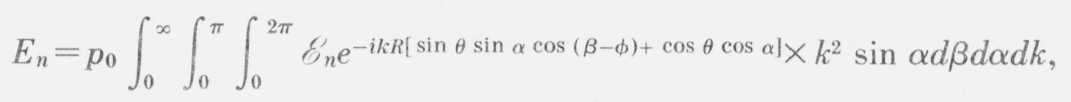

where $(R, \theta, \phi)$ are the spherical coordinates of the observer.

In the special case $n=1$, the $\beta$ integration is effected immediately since $a_{33}$ is independent of $\beta$. Thus, it follows that

$$
E_{1}=\frac{i \mu_{0} \omega p_{0}}{k_{0}^{2}(2 \pi)^{-1}} \nabla^{2} I(R, \theta),
$$

where

$$
I(R, \theta)=k_{0}^{2} \int_{0}^{\infty} \int_{0}^{\pi} \frac{a_{33}}{\Delta} e^{-i k R \cos \theta \cos \alpha} J_{0}(k R \sin \theta \sin \alpha) \sin \alpha d \alpha d k
$$

To evaluate this integral, Mittra and Deschamps [1963] recognize that $I$ may be written as a sum of a singular part $I_{s}$ and a finite part $I_{f}$. That is, $I_{s}$ is of order $(1 / R)$ for $R$ tending to zero, whereas $I_{f}$ is finite for all $R$ and $\theta$. Following some ingenious manipulations, they find that

$$
\nabla^{2} I_{s}=-\frac{\pi}{2}\left(\frac{\epsilon_{0}}{\epsilon}\right)^{1 / 2} \frac{1}{\rho} \frac{\partial}{\partial \rho} \rho \frac{\partial}{\partial \rho} \frac{1}{R^{\prime}},
$$


where $R^{\prime}=\left[\frac{\epsilon}{\epsilon_{0}} z^{2}+\frac{\hat{\epsilon}}{\epsilon_{0}} \rho^{2}\right]^{1 / 2}$. This result has the correct singularity and, in addition, it demon-

strates that $I_{s}$ is independent of $g$, the off-diagonal factor in the expression for the tensor dielectric constant (e.g., (22)).

The evaluation of $\nabla^{2} I_{f}$ appears to be more complicated. It turns out that while $I_{f}$ is finite at the origin, $\nabla^{2} I_{f}$ does have a singularity of the type $O(1 / R)$. Mittra and Deschamps [1963] have given an explicit expression for $\nabla^{2} I_{f}$ but it involves double integrals which, fortunately, have finite limits. Apparently, a great deal of numerical work is required in order to obtain useful numerical results from this approach. It is rather surprising that they do not carry out the $\alpha$ integration explicitly by using contour integration methods. This requires that one evaluate the residues of the poles of the integrand which occur at the zeroes of $\Delta$ for a fixed value of $k$. This operation will not be carried through since it leads directly to the (single) integral representation, given by Barsukov [1959] and Arbel and Felsen [1963], which may be obtained in a more direct fashion as outlined below. The latter approach is particularly appropriate when dealing with horizontally stratified media.

\section{Sources in Stratified Annisotropic Media}

Before tackling the generally stratified medium, the appropriate expressions for the (primary) field of a point source in a homogeneous medium are derived. With respect to the Cartesian coordinate system, an electric dipole is located at $\left(0,0, z_{0}\right)$. The $\mathrm{d}$-c magnetic field is oriented along the $z$ axis and thus the dielectric dyadic $\hat{\epsilon}$ has the tensor form.

$$
(\epsilon)=\left(\begin{array}{ccc}
\epsilon & -i g & 0 \\
i g & \epsilon & 0 \\
0 & 0 & \hat{\epsilon}
\end{array}\right),
$$

where $\epsilon, \hat{\epsilon}$, and $g$ are specified constants for each homogeneous region. For convenience, Maxwell's equations

$$
\vec{\nabla} \times \vec{H}=i \omega \tilde{\epsilon} \cdot \vec{E}+\vec{J}
$$

and

$$
\nabla \times \vec{E}=-i \mu_{0} \omega \vec{H}
$$

are supplemented by the divergence relations

$$
\nabla \cdot(\tilde{\epsilon} \cdot \vec{E})=\bar{\rho}=\frac{i}{\omega} \vec{\nabla} \cdot \vec{J}
$$

and

$$
\nabla \cdot \vec{H}=0
$$

where $\bar{\rho}$ is the charge density.

The preceding equations may be written out explicitly in terms of cylindrical coordinate systems. Then, it is a straightforward matter to eliminate the $E_{r}, E_{\phi}, H_{r}$, and $H_{\phi}$ components. It is found that 


$$
\nabla_{t}^{2} E_{z}+\frac{\hat{\epsilon}}{\epsilon} \frac{\partial^{2} E_{z}}{\partial z^{2}}+\frac{k_{0}^{2} \hat{\epsilon} E_{z}}{\epsilon_{0}}-\frac{\mu_{0} \omega g}{\epsilon} \frac{\partial H_{z}}{\partial z}=i \mu_{0} \omega J_{z}+\frac{1}{\epsilon} \frac{\partial \bar{\rho}}{\partial z}
$$

and

$$
\nabla_{t}^{2} H_{z}+\frac{\partial^{2} H_{z}}{\partial z^{2}}+\frac{k_{0}^{2}}{\epsilon \epsilon_{0}}\left(\epsilon^{2}-g^{2}\right) H_{z}+\frac{\hat{\epsilon}}{\epsilon} \omega g \frac{\partial E_{z}}{\partial z}=-[\vec{\nabla} \times \vec{J}]_{z}+\frac{\omega g \bar{\rho}}{\epsilon}
$$

where

$$
[\vec{\nabla} \times \vec{J}]_{z}=\frac{\partial J_{\phi}}{\partial \rho}-\frac{1}{\rho} \frac{\partial J_{\rho}}{\partial \phi}
$$

and

$$
\nabla_{t}^{2}=\frac{1}{\rho} \frac{\partial}{\partial \rho} \rho \frac{\partial}{\partial \rho}+\frac{1}{\rho^{2}} \frac{\partial}{\partial^{2} \phi}
$$

is the transverse Laplacian operator. In this section $k_{0}=\omega / c=2 \pi /$ wavelength and $\epsilon_{0}$ is the dielectric constant of free space.

The above coupled equations for $E_{z}$ and $H_{z}$ will hold for any (piecewise) homogeneous region. A subscript $m$ is added to the field quantities when reference is to be made to the $m$ th homogeneous region. The integer $m$ is allowed to range from 1 to $M$. The source dipole is taken to be in the $p$ th layer where $p$ is an integer equal to or between 1 and $M$.

To facilitate matching of boundary conditions, it is convenient to employ the following spectral representations of Dirac delta functions:

and

$$
\delta\left(z-z_{0}\right)=\frac{1}{2 \pi} \int_{-\infty}^{+\infty} e^{i u\left(z-z_{0}\right)} d u
$$

$$
\frac{\delta(\rho)}{\rho}=\int_{0}^{\infty} J_{0}(\lambda \rho) \lambda d \lambda \text {. }
$$

Therefore, if the dipole of moment $p_{0}$, located at $z=z_{0}$, is oriented in the $z$ direction the current density has the following form:

$$
J_{z}=i \omega p_{0} \delta\left(z-z_{0}\right) \delta(\rho) /(2 \pi \rho)=\frac{i \omega p_{0}}{(2 \pi)^{2}} \int_{0}^{\infty} \int_{-\infty}^{+\infty} J_{0}(\lambda \rho) e^{i u\left(z-z_{0}\right)} \lambda u d \lambda d u .
$$

The corresponding formula for the charge density is

$$
\bar{\rho}=-\frac{i p_{0}}{(2 \pi)^{2}} \int_{0}^{\infty} \int_{-\infty}^{+\infty} J_{0}(\lambda \rho) e^{i u\left(z-z_{0}\right)} \lambda u d \lambda d u .
$$

In the $s$ th region, which contains the source, the axial field components are written in the form

$$
E_{z}=E_{z}^{p}+E_{z}^{s}
$$

and

$$
H_{z}=H_{z}^{p}+H_{z}^{s}
$$

where the primary and secondary fields are indicated by a superscript $p$ and $s$, respectively. It is understood that, if the sth region were unbounded, the secondary fields would not be present.

Taking a clue from the appearance of (75) and (76), the axial components of the primary field are written in the form

and

$$
E_{z}^{p}=\frac{p_{0}}{(2 \pi)^{2}} \int_{0}^{\infty} \int_{-\infty}^{+\infty} A(\lambda, u) J_{0}(\lambda \rho) e^{i u\left(z-z_{0}\right)} d \lambda d u
$$

$$
H_{z}^{p}=\frac{p_{0}}{(2 \pi)^{2}} \int_{0}^{\infty} \int_{-\infty}^{+\infty} B(\lambda, u) J_{0}(\lambda \rho) e^{i u\left(z-z_{0}\right)} d \lambda d u
$$


Substituting (75), (76), (79), and (80) into (69) and (70), explicit expressions may be found for the functions $A$ and $B$. The integrations, with respect to $u$, may be easily carried out and the final result is

$$
E_{z}^{p}=\frac{\epsilon}{\hat{\epsilon}^{2}} p_{0} \int_{0}^{\infty}\left[u_{1}\left(u_{1}^{2}+s_{1}^{2}\right) e^{-u_{1}\left|z-z_{0}\right|}-u_{2}\left(u_{2}^{2}+s_{1}^{2}\right) e^{-u_{2}\left|z-z_{0}\right|}\right] \times \frac{J_{0}(\lambda \rho)}{s_{2}^{2}\left(u_{2}^{2}-u_{1}^{2}\right)} \lambda^{3} d \lambda
$$

and

$$
H_{z}^{p}=-\frac{\omega g}{\hat{\epsilon}} p_{0} \frac{\left(z-z_{0}\right)}{\left|z-z_{0}\right|} \int_{0}^{\infty}\left[e^{-u_{1}\left|z-z_{0}\right|}-e^{-u_{2}\left|z-z_{0}\right|}\right] \times \frac{J_{0}(\lambda \rho) \lambda^{3} d \lambda}{u_{2}^{2}-u_{1}^{2}}
$$

where

$$
s_{1}^{2}=k_{0}^{2}\left(\epsilon / \epsilon_{0}\right)-\lambda^{2}, s_{2}^{2}=(\epsilon / \hat{\epsilon})\left[k_{0}^{2}\left(\hat{\epsilon} / \epsilon_{0}\right)-\lambda^{2}\right]
$$

where $u_{1}$ and $u_{2}$ are roots of the equation

$$
u^{4}+u^{2}\left(s_{1}^{2}+s_{2}^{2}\right)+s_{1}^{2} s^{2}-s_{2}^{2} \frac{k_{0}^{2} g^{2}}{\epsilon \epsilon_{0}}=0
$$

chosen so that $\operatorname{Re} u_{1}>0$ and $\operatorname{Re} u_{2}>0$.

Expressions for the other components of the primary field may be readily found from the axial components given here.

Expressions for the secondary field, in the sth region, are now sought in the form

$$
\begin{aligned}
E_{z}^{s}=\frac{\epsilon}{\hat{\epsilon}^{2}} p_{0} \int_{0}^{\infty}\left\{u_{1}\left(u_{1}^{2}+s_{1}^{2}\right)\left[\varphi_{1}(\lambda) e^{-u_{1} z}+\psi_{1}(\lambda) e^{+u_{1} z}\right]\right. & \\
& \left.+u_{2}\left(u_{2}^{2}+s_{1}^{2}\right)\left[\varphi_{2}(\lambda) e^{-u_{2} z}+\psi_{2}(\lambda) e^{+u_{2} z}\right]\right\} \frac{J_{0}(\lambda \rho) \lambda^{3} d \lambda}{s_{2}^{2}\left(u_{2}^{2}-u_{1}^{2}\right)}
\end{aligned}
$$

and

$$
H_{z}^{s}=-\frac{\omega g}{\hat{\epsilon}} p_{0} \int_{0}^{\infty}\left\{\left[\varphi_{1}(\lambda) e^{-u_{1} z}-\psi_{1}(\lambda) e^{+u_{1} z}\right]-\left[\varphi_{2}(\lambda) e^{-u_{2} z}-\psi_{2}(\lambda) e^{+u_{2} z}\right]\right\} \frac{J_{0}(\lambda \rho) \lambda^{3} d \lambda}{\left(u_{2}^{2}-u_{1}^{2}\right)}
$$

where the functions $\varphi_{1}(\lambda), \varphi_{2}(\lambda), \psi_{1}(\lambda)$, and $\psi_{2}(\lambda)$ are undetermined.

The axial field components, $E_{z}^{n}$ and $H_{z}^{n}$, in the other homogeneous slabs would have the same form as the above expressions if the appropriate material constants of the medium are employed (e.g., for the $n$th slab $\epsilon, \hat{\epsilon}$, and $g$ are replaced by $\epsilon_{n}, \hat{\epsilon}_{n}$, and $g_{n}$, respectively). In each slab, there are four unknown functions (i.e., $\varphi_{1}^{n}, \varphi_{2}^{n}, \psi_{1}^{n}$, and $\psi_{2}^{n}$ ), and these may be found from the matching of the four tangential field components at each interface. In addition, it is required that only outgoing waves are permitted at $z= \pm \infty$. Thus, in principle, the complete formal solution is available.

Using the method outlined here, Barsukov [1959] has given an explicit expression for the field of a vertical electrical dipole at $z=z_{0}$, which is immersed in a semi-infinite anisotropic medium $(z>0)$. In the region below (i.e., $z<0$ ), the medium is assumed to be free space with electrical constants $\epsilon_{0}$ and $\mu_{0}$.

For $z>0$, the axial fields have the form

$$
\begin{aligned}
& E_{z}=E_{z}^{p}+E_{z}^{s}, \\
& H_{z}=H_{z}^{p}+H_{z}^{s} .
\end{aligned}
$$

Here, $E_{z}^{p}$ and $H_{z}^{p}$ are the primary fields given by (81) and (82), while $E_{z}^{s}$ and $H_{z}^{s}$ are the secondary 
fields which have the form of (85) and (86) with the condition that $\psi_{1}(\lambda)$ and $\psi_{2}(\lambda)$ are both zero.

For the isotropic region $z<0$, it is convenient to represent the field in terms of electric and magnetic Hertz vectors, $\vec{\Pi}_{e}$ and $\vec{\Pi}_{m}$. Thus

$$
\begin{aligned}
& \vec{E}=k_{0}^{2} \vec{\Pi}_{e}+\nabla\left(\nabla \cdot \vec{\Pi}_{e}\right)-i \mu_{0} \omega \nabla \times \vec{\Pi}_{m}, \\
& \vec{H}=i \epsilon_{0} \omega \nabla \times \vec{\Pi}_{e}+k_{0}^{2} \vec{\Pi}_{m}+i \epsilon_{0} \omega \nabla \times \vec{\Pi}_{e},
\end{aligned}
$$

where

$$
\vec{\Pi}_{e}=\left(0,0, \Pi_{e}\right) \text { and } \vec{\Pi}_{m}=\left(0,0, \Pi_{m}\right)
$$

The following representations are employed:

$$
\Pi_{e}=\frac{p_{0}}{k_{0}} \int_{0}^{\infty} F_{1}(\lambda) J_{0}(\lambda \rho) e^{u_{0} z} \lambda d \lambda
$$

and

$$
\Pi_{m}=\frac{i p_{0}}{k_{0}} \int_{0}^{\infty} F_{2}(\lambda) J_{0}(\lambda \rho) e^{u_{0} z} \lambda d \lambda
$$

where $u_{0}=\left(\lambda^{2}-k_{0}^{2}\right)^{1 / 2}$ with $\operatorname{Re} u_{0}>0$. The scalar functions, $\Pi_{e}$ and $\Pi_{m}$, satisfy the wave equation $\left(\nabla^{2}+k_{0}^{2}\right) \Pi_{e, m}=0$; they behave as outgoing waves at $z=-\infty$, and they are independent of the azimuthal coordinate $\phi$. The functions $\varphi_{1}(\lambda), \varphi_{2}(\lambda), F_{1}(\lambda)$, and $F_{2}(\lambda)$ can be determined explicitly from the four boundary conditions (requiring the continuity of the tangential fields) at $z=0$. Barsukov [1959] gives

$$
\begin{aligned}
& \varphi_{1}\left(u_{1}, u_{2}\right)=-\frac{1}{\delta\left(u_{1}, u_{2}\right)}\left[\delta\left(-u_{1}, u_{2}\right) e^{-u_{1} z_{0}}+2 \frac{\epsilon}{\hat{\epsilon}} u_{2} \lambda^{2}(\hat{\boldsymbol{\epsilon}}-\epsilon)\left(u_{2}^{2}+s_{1}^{2}\right) e^{-u_{2} z_{0}}\right] . \\
& \varphi_{2}\left(u_{1}, u_{2}\right)=\varphi_{1}\left(u_{2}, u_{1}\right) \\
& F_{1}\left(u_{1}, u_{2}\right)=\frac{2 \epsilon k_{0}}{\hat{\epsilon} \delta\left(u_{1}, u_{2}\right)}\left[u_{1}\left(u_{1}^{2}+s_{1}^{2}\right)\left(u_{0}+u_{2}\right) e^{-u_{1} z_{0}}-u_{2}\left(u_{2}^{2}+s_{1}^{2}\right)\left(u_{0}+u_{1}\right) e^{-u_{2} z_{0}}\right], \\
& F_{2}\left(u_{1}, u_{2}\right)=\frac{2 i g k_{0}^{2}}{\hat{\epsilon} \delta\left(u_{1}, u_{2}\right)}\left[u_{1}\left(\epsilon u_{1} u_{2}-\epsilon_{0} s_{2}^{2}\right) e^{-u_{1} z_{0}}-u_{2}\left(\epsilon u_{1} u_{2}-\epsilon_{0} s_{2}^{2}\right) e^{-u_{2} z_{0}}\right] \text {, }
\end{aligned}
$$

where

$$
\delta\left(u_{1}, u_{2}\right)=\left(u_{2}-u_{1}\right)\left[\epsilon_{0} s_{2}^{2}\left(u_{1} u_{2}-s_{1}^{2}\right)-\epsilon u_{0}^{2}\left(u_{1} u_{2}-s_{2}^{2}\right)+u_{0}\left(u_{1}+u_{2}\right)\left(\epsilon_{0} s_{2}^{2}-\epsilon u_{1} u_{2}\right)\right] .
$$

This constitutes the complete formal solution of the problem. Explicit expressions for the field components are obtained by differentiation according to (89), (90), (91), and (92).

The general results are rather complicated; however, some simplicity is achieved by considering the radiation fields in the lower half space. Physically, this corresponds to the situation where the observer is at a distance, below the interface, which is large compared with the wavelength. The integrals to contend with are of the form

$$
\int_{0}^{\infty} A(\lambda) J_{0}(\lambda \rho) e^{-u\left|z_{0}\right|} \lambda d \lambda=I(\rho, z)
$$

where $A(\lambda)$ is a slowly varying function of $\lambda$. A saddle point evaluation of the integral yields

$$
I(\rho, z) \sim i k_{0} \cos \theta A\left(k_{0} \sin \theta\right) e^{-i k_{0} R} / R
$$


where

$$
R=\left(\rho^{2}+z^{2}\right)^{1 / 2} \text { and } \tan \theta=-(\rho / z)
$$

As indicated, the saddle point or principal value occurs at $\lambda=k_{0} \sin \theta$ where $\theta$ is the angle subtended by the direction to the observer and the negative $z$-axis. This asymptotic result is valid provided $\left|k_{0} z\right|>>1$ and $|z|>>z_{0}$. The first inequality appears to be very important since if $k z$ is small, the contributions from the poles of $A(\lambda)$ lead to surface waves which have a significant amplitude compared with the space wave. The pole wave contributions were not considered by Barsukov [1959].

\section{Radiation Fields in Ānisotropic Media}

An important aspect of this subject is the nature of the radiation or far-zone fields when both source and observer are within the anisotropic medium. This particular problem has been considered in some detail by Arbel and Felsen [1963]. In their work they make extensive use of the geometrical properties of the refractive index surfaces. For purposes of this discussion, the medium will be considered homogeneous and unbounded. Furthermore, the source is an electric dipole of moment $p_{0}$ located at the origin of a cylindrical coordinate system $(\rho, \phi, z)$ and oriented in the $z$ direction. The applied d-c magnetic field is also directed in the $z$ direction. The medium is nonmagnetic with permeability $\mu_{0}$, while the electric properties are described in terms of a tensor dielectric constant $\tilde{\epsilon}$ of the form given by (22). Losses in the medium are assumed to be zero. The Fourier-Bessel representations of the fields given by Arbel and Felsen [1963] are closely related to (81) and (82) first given by Barsukov [1959]. The essential modifications to (81) and (82) are to replace the variable $\lambda$ by $k_{0} S$ where $S$ is now a dimensionless variable and $k_{0}=\omega / c$. By using identities $J_{0}(x)=\frac{1}{2}\left[H_{0}^{(1)}(x)+H_{0}^{(2)}(x)\right]$ and $H_{0}^{(2)}(x)=-H_{0}^{(1)}(-x)$, the integration contour may be changed from along the positive real axis to a straight line running from $S=-\infty$ to $+\infty$. Because of a branch point singularity at $S=0$, the integration path is indented around this point by a small semi-circle in the lower half plane of the complex $S$ plane. Additional substitutions in (81) and (82) are to replace the axial propagation constants $u, u_{1}$, and $u_{2}$ by $i k_{0} C, i k_{0} C_{1}$, and $i k_{0} C_{2}$, respectively. The subscripts 1 and 2 may be identified with the ordinary and the extraordinary waves in the classical magneto-ionic theory.

The resulting vector electric fields may be written in the form

$$
\vec{E}=\overrightarrow{E_{1}}+\overrightarrow{E_{2}}
$$

where the field components are given by

$$
\begin{gathered}
\left(E_{\rho}\right)_{1,2}=i A \frac{z}{|z|} \int_{-\infty}^{+\infty} S^{2} \frac{S^{2} \mp \Delta}{ \pm \Delta} H_{1}^{(2)}\left(k_{0} S \rho\right) \exp \left[-i k_{0} C_{1,2}|z|\right] d S \\
\left(E_{\phi}\right)_{1,2}=2 A \frac{\epsilon^{\prime}}{\epsilon-1} \int_{-\infty}^{+\infty} \frac{S^{2}}{ \pm \Delta} H_{1}^{(2)}\left(k_{0} S \rho\right)\left[-i k_{0} C_{1,2}|z|\right] d S
\end{gathered}
$$

and

$$
\left(E_{z}\right)_{1,2}=A \int_{-\infty}^{+\infty} \frac{S^{2} \mp \Delta}{ \pm \Delta} \frac{C_{1,2} S^{3}}{1-S^{2}} H_{0}^{(2)}\left(k S_{\rho}\right) \exp \left[-i k_{0} C_{1,2}|z|\right] d S
$$

where

$$
A=\mu_{0} \omega^{2} p_{0} /(16 \pi)
$$

and

$$
\Delta=\frac{\epsilon-\epsilon_{0}}{\left|\epsilon-\epsilon_{0}\right|}\left[S^{4}+4\left(\frac{g}{\epsilon-1}\right)^{2}\left(1-S^{2}\right)\right]^{1 / 2}
$$


The functions $C_{1}$ and $C_{2}$ are proportional to $u_{1}$ and $u_{2}$ as noted. The latter may be obtained from the roots of the quadratic equation in $u^{2}$ given by (84), which is equivalent to the AppletonHartree equation. Explicitly,

$$
C_{1,2}=\left[U \pm\left(U^{2}-W\right)^{1 / 2}\right]^{1 / 2}=\left[U \pm\left(\frac{\epsilon}{\epsilon_{0}}-1\right) \frac{\Delta}{2}\right]^{1 / 2}
$$

where

$$
\begin{gathered}
U=\frac{\epsilon}{\epsilon_{0}}-\frac{\left(\epsilon+\epsilon_{0}\right)}{2 \epsilon_{0}} S^{2}, \\
W=\frac{\epsilon}{\epsilon_{0}}\left(1-S^{2}\right)\left[\frac{\epsilon^{2}-g^{2}}{\epsilon \epsilon_{0}}-S^{2}\right] .
\end{gathered}
$$

Arbel and Felsen [1963] have devoted a great deal of attention to the proper definition of the multivalued function $C$ and the disposition of the integration path in relation to the singularities of the integrands in (100), (101), and (102). In the first place, it is to be observed that $C_{1}$ and $C_{2}$ are associated respectively with the + and - signs in (105), and that $\left(U^{2}-W\right)^{1 / 2}$ is defined to be positive when real. The definition of $C_{1}$ given above implies that $C_{1}$ has branch-point singularities at $U^{2}=W$ (or at $\Delta=0$ ), and at those values of $S$ for which $C_{1}=0$. Similarly, $C_{2}$ has branch-point singularities at $\Delta=0$, and at those values of $S$ for which $C_{2}=0$. However, a series expansion of the total integrand about $\Delta=0$ contains only even powers of $\Delta$, which means that $\Delta=0$ is a regular point. Thus, no special care need be taken in the definition of $C_{1,2}$ at $\Delta=0$ if the "ordinary" and "extraordinary" are combined and treated together. Also, it should be mentioned that the factor $\left(1-S^{2}\right)^{-1}$ in the integrand of (102) does not give rise to a pole contribution since it is canceled by other factors.

If $C_{1}$ or $C_{2}$ are real, the quantity $\exp \left(-i k_{0} C_{1,2} z\right)$ represents a propagating plane wave in the positive $z$-direction away from the source. On physical grounds, it may be supposed that each component plane wave, in the total spectrum, has a positive component in the $z$-direction. This radiation condition permits the unique determination of $C_{1,2}$ over the range of $S$ for which the propagation constants are real. As Arbel and Felsen [1963] show, the contributions to the power flow, when the propagations are complex, are zero. The resulting "energy radiation condition" is that, when $C_{1,2}$ is real,

$$
\left(\frac{\mu_{0}}{\epsilon_{0}}\right)^{1 / 2} Y_{1,2}=C_{1,2} \frac{4}{\left(\Delta^{2} \pm S^{2} \Delta\right)}\left(\frac{g}{\epsilon-1}\right)^{2}>0
$$

where $Y_{1}$ and $Y_{2}$ may be interpreted as admittances for ordinary and extraordinary waves traveling in the positive $z$-direction.

It is evident from the above inequality that the sign of $C_{1,2}$ is identical with that of $\Delta^{2}-S^{2} \Delta$. This condition for real $C_{1,2}$, when supplemented with the condition $\operatorname{Im} C_{1,2}<0$ for $C_{1,2}$ complex, may be used to determine the analytic continuation of the function $C_{1,2}(S)$ around branch-point singularities on the real axis of the $S$ plane.

As indicated above, the propagating modes correspond to the situation where

$$
C_{1,2}^{2}=U \pm\left(U^{2}-W\right)^{1 / 2}
$$

is positive real. Thus, it is evident that ordinary and extraordinary modes propagate when $U>0$. On the other hand, if $\left(U^{2}-W\right)>|U|$, i.e., $W<0$, the ordinary mode propagates for $U \lessgtr 0$, while the extraordinary mode does not propagate.

There is actually a very simple graphical procedure which utilizes the dispersion curves of $C(S)$ versus $S$. For example, the plane wave, characterized by the variation

$$
\exp \left[-i k_{0}(S \rho+C z)\right]
$$


carries power in the direction perpendicular to the dispersion curve at the point $S, C$. The radiation condition corresponding to choosing those values of $C_{1}$ and $C_{2}$ for which the outward normal to the dispersion curve, has a positive component in the $z$-direction.

The integrals in (100), (101), and (102) may be evaluated asymptotically by the method of saddle points. This has been demonstrated by Arbel and Felsen [1963] in an elegant manner.

It is convenient to introduce spherical coordinates $(r, \theta)$ which are defined by $\rho=r \sin \theta$ and $z=r \cos \theta$. Then, under the assumption that $k_{0} r \sin \theta>>1$, the asymptotic approximation

$$
H_{n}^{(2)}\left(k_{0} S \rho\right) \sim\left(\frac{2}{\pi k_{0} S r \sin \theta}\right)^{1 / 2} \exp \left[-i k_{0} S r \sin \theta+i n \pi / 2+i \pi / 4\right]
$$

is introduced. Thus, for most practical purposes, the integrals to contend with are of the form

where

$$
B=\int_{-\infty}^{+\infty} L(S) \exp \left[-i k_{0} r M(S)\right] d S
$$

$$
M(S)=C(S)|\cos \theta|+S \sin \theta
$$

and $k_{0} r$ is to be regarded as a large parameter.

The principal contributions to the integrand occur at the vicinity of the saddle points $S^{i}$ determined by

$$
M^{\prime}\left(S^{i}\right)=0,
$$

or

$$
C^{\prime}\left(S^{i}\right)=-|\tan \theta| \text {. }
$$

Propagating waves correspond to the saddle points where both $C\left(S^{i}\right)$ and $S^{i}$ are real. If these quantities are complex, the associated fields are highly attenuated and need not concern us further.

The technique to evaluate the integral asymptotically consists of deforming the integration path to the steepest descent paths through each saddle point. The ends of these paths are connected up in the valley regions of the $S$ plane where $-\operatorname{Im} C(S)$ is a large positive quantity. Thus, because of the heavy exponential damping, these portions of the path contribute a negligible amount and may be ignored for the present analysis. In the vicinity of a real saddle point $S_{i}$, the factor $\exp \left[-i k_{0} r C(S)\right]$ decays most rapidly along the steepest descent path which is inclined at angles of $\pm 45^{\circ}$ with the real axis.

The asymptotic representation for the integral is then found to be of the form

$$
B \cong \sum_{i} B^{(i)}
$$

where the contribution from an individual saddle point is

$$
B^{(i)} \sim L\left(S^{i}\right)\left[\frac{2 \pi}{k_{0} r\left|M^{\prime \prime}\left(S^{i}\right)\right|}\right]^{1 / 2} e^{\mp i \pi / 4} e^{-i k_{0} r M\left(S^{i}\right)}
$$

where the argument $-i \pi / 4$ is used when $M^{\prime \prime}\left(S^{i}\right)>0$, and $+i \pi / 4$ is used when $M^{\prime \prime}\left(S^{i}\right)<0$. It is assumed that the integrals are of the form such that the saddle points have alternating signs along the real axis of $S$.

To express the final results for the radiation fields, the unit vectors, $\hat{\rho}, \hat{\phi}$, and $\hat{z}$ are introduced along with the polarization vectors

$$
\overrightarrow{p^{i}}=\hat{\rho} S^{i}+\hat{z} C^{i} \text { and } \vec{q}_{i}=\hat{\rho} S^{i}+\hat{z} \frac{1-\left(S^{i}\right)^{2}}{C^{i}}
$$


Then, following Arbel and Felsen [1963], it is found that the asymptotic versions of (100), (101), and (102) may be written

$$
\hat{\rho} E_{\rho}+\hat{z} E_{z} \sim 2 i A \sum_{i} \beta^{i}\left(R^{i}\right)^{1 / 2} G^{i} \frac{e^{-i k_{0} r N i(\theta)}}{k_{0} r}(\vec{q} \times \hat{\phi}),
$$

and

$$
E_{\phi} \sim 2 A \sum_{i} \beta^{i}\left(R^{i}\right)^{1 / 2} F^{i} \frac{e^{-i k_{0} r N i(\theta)}}{k_{0} r}
$$

where $N_{i}(\theta)$ is the "ray refractive index" defined by

$$
M\left(S^{i}\right)=N^{i}(\theta)
$$

The radius of curvature $R^{i}$ of the dispersion curve (i.e., $C(S)$ versus $S$ for real $S$ ) at the saddle point $S^{i}$ is obtained from

$$
R^{i}=\frac{\left\{1+\left[C^{\prime}\left(S^{i}\right)\right]^{2}\right\}^{3 / 2}}{\left|C^{\prime \prime}\left(S^{i}\right)\right|}=\left\{\left|C^{\prime \prime}\left(S^{i}\right)\right| \cos ^{3} \theta\right\}^{-1}
$$

In (118) and (119) $\beta^{i}=1$ if $M^{\prime \prime}\left(S^{i}\right)<0$ and $\beta^{i}=i$ if $M^{\prime \prime}\left(S^{i}\right)>0$. Finally, the amplitude factors are obtained from

$$
F^{i}=\left(\frac{S_{i}}{\sin \theta}\right) \frac{2 S^{i}}{\Omega}\left(\frac{g}{\epsilon-\epsilon_{0}}\right) \cos \theta
$$

and

$$
G^{i}= \pm\left(\frac{S_{i}}{\sin \theta}\right)^{1 / 2} \frac{C^{i} S^{i}}{1-\left(S^{i}\right)^{2}} \frac{\left(S^{i}\right)^{2}-\Omega}{\Omega} \cos \theta
$$

where

$$
\Omega=\frac{2\left[C^{2}\left(S^{i}\right)-\frac{\epsilon}{\epsilon_{0}}+\frac{\epsilon+\epsilon_{0}}{2 \epsilon_{0}}\left(S^{i}\right)^{2}\right]}{\left(\frac{\epsilon}{\epsilon_{0}}-1\right)} .
$$

It is understood that (118) and (119) both hold individually for ordinary and extraordinary waves. The summations extend over all contributing saddle points in each case. The exponential factors involve the ray refractive inaex $N^{i}(\theta)$ which is related to the polarization vector $p_{i}=\hat{p} S^{i}+\hat{z} C^{i}$ by the scalar identity $N^{i}(\theta)=\vec{p} \cdot \overrightarrow{r_{0}}$ where $\overrightarrow{r_{0}}$ is a unit vector parallel to the ray direction which points from the source to the observer.

For the results given above, it is specifically assumed that $\hat{\epsilon} / \epsilon_{0}>0$. If $\hat{\epsilon} / \epsilon_{0}<0$, then the relevant field expressions are obtained by making the substitutions

$$
(\hat{\epsilon})^{1 / 2} \rightarrow-i(-\hat{\epsilon})^{1 / 2}, C \rightarrow i C, \text { and } S \rightarrow i S .
$$

The characteristics of the radiation field in an anisotropic medium are critically dependent on the shape of the dispersion curve (or refractive index surface). It is evident that the number of contributing rays and their amplitude may be determined by a careful examination of the dispersion curve. The presence of open branches on the dispersion curve indicates presence of shadows where no rays may propagate. However, the field in the shadow region may be finite as a result of diffraction [Brekhovskikh, 1960]. This aspect of the subject is outside the scope of the present survey. Also, the presence of points of inflection in the dispersion curve (i.e., $\left.C^{\prime \prime}\left(S^{i}\right) \cong 0\right)$ will lead 
to focusing effects which may be described by a modified saddle point procedure involving Airy functions [Wait, 1962]. This topic is also outside the scope of this discussion. However, the interested reader may find an elegant treatment of this particular problem in the paper by Arbel and Felsen [1963].

\section{Final Remarks}

The present expository paper has been a self-contained treatment of the theory of radiation from sources in cold magnetoplasma media.

In this review, no mention has been made of antenna impedance for anisotropic media. While this would seem to be a natural topic for this discussion, it has been deferred until a later time. One reason for this is the need to consider thermal effects and sheath phenomena, which are important near the source. Consideration resulting from the finite temperature leads to contributions from the acoustic-type waves not usually considered part of magneto-ionic theory.

I am grateful to Dr. K. Furutsu for making a number of corrections in the manuscript. I also thank Mrs. Eileen Brackett for help in preparing this material.

\section{References}

Ament, W. S. (Feb. 1963), On the electromagnetic Green's function for a homogeneous anisotropic medium and its application for antenna theory, Contract NAS5-585, Scientific Report No. 4, Electromagnetic Research Corporation.

Arbel, E., and L. B. Felsen (1963), On the radiation from sources in anisotropic media, Part I, pp. 391-420 and Part II, pp. 421-459, in Electromagnetic Theory and Antennas, ed. by E. C. Jordan (Pergamon Press, Oxford).

Barsukov, K. A. (Nov. 1959), Radiation of electromagnetic waves from a point source in a gyrotropic medium with a separation boundary, Radiotekhnika i Elektronika 4, 1759-1764.

Brekhovskikh, L. M. (1960), Waves in layered media (Academic Press Ltd., New York).

Bunkin, F. V. (Feb. 1957), On radiation in anisotropic media, J. Exptl. Theoret. Phys. USSR 32, 338-346.

Kogelnik, H. (Sept.-Oct. 1960), On electromagnetic radiation in magneto-ionic media, J. Res. NBS 64D (Radio Prop.), No. 5, 515-523.

Kuehl, H. H. (Sept. 1962), Electromagnetic radiation from an electric dipole in a cold anisotropic plasma, The Physics of Fluids 5, 1095-1103.

Mittra, R., and G. A. Deschamps (1963), Field solution for a dipole in an anisotropic medium, pp. 495-512 in Electromagnetic Theory and Antennas (ed. by E. C. Jordan), Pergamon Press, Oxford.

Meecham, W. C. (Dec. 1961), Source and reflection problem in magneto-ionic media, Physics of Fluids 4, $1517-1524$.

Wait, J. R. (1962), Electromagnetic waves in stratified media (Pergamon Press, Oxford, and Macmillan Co., New York).

\section{9. Ādditional References}

Abraham, L. G. Jr. (Aug. 1953), Extensions, including antenna radiation and plane wave scattering, Rep. No. 13, Cornell School of Electrical Engineering, Ithaca, New York.

Clemmow, P. C. (1963), On the theory of radiation from a source in a magneto-ionic medium, pp. 461-475 in Electromagnetic Theory and Antennas (ed. by E. C. Jordan), Pergamon Press, Oxford.

Ford, G. W. (Nov. 1961), Electromagnetic radiation from a source in a plasma, Annals of Physics 16, 185-200.

Kogelnik, H., and H. Motz (1963), Electromagnetic radiation from sources in an infinite anisotropic medium and the significance of the Poynting vector, pp. 477-493 in Electromagnetic Theory and Antennas (ed. by E. C. Jordan), Pergamon Press, Oxford.

Wait, J. R. (Dec. 1960), Propagation of electromagnetic waves along a thin plasma sheet, Can. J. Phys. 38, $1586-1594$.

Wait, J. R. (1961), The electromagnetic fields of a dipole in the presence of a thin plasma sheet, Appl. Sci. Res. Sec. B, 8, 397-417.

(Paper 68B3-125) 